\title{
A Portable Fuzzy Sink Scheme for Wireless Sensor Network Life Expectancy Enhancement
}

\author{
Wasswa Shafik ${ }^{1 *}$, and S. Mojtaba Matinkhah ${ }^{2}$ \\ 1 Computer Engineering Department ${ }^{1}$; Yazd University; Yazd, Iran; wasswashafik@gmail.com \\ 2 Computer Engineering Department 1; Yazd University; Yazd, Iran; matinkhah@yazd.ac.ir \\ * Correspondence: wasswashafik@gmail.com; Tel.: +256 700654948, +98 9132667699, +98 9334701475
}

\begin{abstract}
Wireless Sensor Networks (WSNs) is considered to be a promising research area since it entails sensing capabilities. WSNs are confronted by fabricated explosion injection attacks and in return results into draining of vitality on sensor nodes routing mainly because of increased capabilities in a broad range of applications where sensor nodes use a battery as the power source. In this paper, we reveal and claim a state-of-the-art model to minimize vitality depletion of nodes within the network in return increases network period to more realistic stretch. It is revealed that the main cause is vitality consumption unbalances in different parts of the network direct resulting from having a static sink. More still, the network nodes neighboring the sink insistently partake in progressing data of other nodes to the sink. Consequently, the network nodes are supplementarily subjected to untimely vitality exhaustion. This paper mainly provides a novel movable (mobile) fuzzy logic sink approach and an intelligent system like expect systems that are able to scrutinize network situation, and make the superlative choice for spreading the network period robotically.
\end{abstract}

Keywords: Mobile sink; Network period; Funneling Effect; Wireless Sensor Network; Vitality Management.

\section{Introduction}

Wireless sensor networks (WSNs) entails clusters of sparsely isolated with devoted sensors used mainly to monitor and record the current condition or (conditions) of the environment, organize the available collectable and collected data to sink location like for instance chief location and the applications are viewed in several physical phenomena including scientific, surveillance, health, and military applications [1]. WSNs have been emerging in computing in the last couple of years witnessed due to the dynamic technological advances in micro electro mechanical systems. There are sensor nodes that a tiny gadget entails data constituent recognition for data attainment from current situation, storage management during local data processing plus data transfer capacities [2].

Several studies related to WSN period expectancy are mushrooming due to increased daily vitality depletion. WSN tend to operate in a low vitality depletion computing and further communicate data via wireless connectivity's transmission. Purposely, their chief duty is receiving data as sensors then there are (data) transmitted via the network [3]. Besides, a power supply circuit distributes energies desirable using device to achieve the automated mission. This power supply circuit regularly contains a battery with partial initial desired-vitality. The sensor network is met to have a prolonged time span sufficient in accomplishing a given needed requirements with low and reduced complexities [4].

A Sensor node is devoted with sensors which sense and receives the mandatory information. Furthermore, to the more sensors, each node in a sensor setup is archetypally equipped through the radio transceiver, it important to that some their wireless communications device or antenna that is used to interconnect or communicate with other neighbor nodes [5], [6]. Nevertheless, as a consequence of the increased vitality distribution from sensor nodes, encompassing the period of WSNs have been an elementary aiming at substantial amount within the past decades within the 
research arena. Most importantly, the time span given a network is categorically known to be operational time of the network set up during through which it can perform the desired operation(s).

Throughout the studies, several challenges in WSN have been revealed mainly among which they include deployment, localization, identity-organization, triangulation and regulator, reportage, vitality-preservation, lastly on this list data progression. Depending on the atmosphere, it is common for sensor nodes to be fault and unreliable as was illustrated in details in [8]. Subsequently, because of the vitality source restraint where communication that overwhelm power punter within nodes, the broadcast per distance of nodes are inadequate for vitality drain mitigations resolves via the application of adaptive fuzzy vitality measures. Practically the nodes that have capacities of sensing are sparsely located far as the sink nodes utilize multi-hop forwarding in delivering data (collectable and collected) to the sink [9].

There exists a thin line between Internet of Things (IoT) and the WSN. The IoTs generally exists at a higher level than WSN. For simplicity, the WSNs are repeatedly a technology based within a mesh setup (physical display), that may be utilized in individual gathering of collectable and collected data and send it via a router to the IoT networks. In general, the WSN consists of wireless sensors, communication (Multi-hop) outcomes unbalanced vitality expenditure in the diverse chunks of the net-work; protuberances like nodes all over the sink diminish their vitality much are considered faster as compared to detached nodes. In case the collectable and sensed collected data may not be magnificently conveyed sink linked, at that moment the retro per sensors adjacently linked to sink converts to logjam for the net epoch [10].

Within the paper, we claim that using a single or multiple mobile sinks (s) based on fuzzy logic for placing these sinks is one of an effective ways through which balancing of the vitality outflow with increased period (network time span) of the network. Notably, Fuzzy logic is an authoritative technique with the ability to deal with decision-making procedures and human reasoning. Intelligent systems like the fuzzy expert system use fuzzy logic in its place of doubled logic. For clarity, an expert scheme of fuzzy characteristics is a collection that depicts member-ship functions (MF) with rules used in deciding data manipulation. Dissimilar to all the earlier approaches, we assume several significant parameters instantaneously to cater during decision making about change location of mobile sink, then the design fuzzy system for a select best new location is availed.

A somatic net node is a vigorous electric device fond of a net with the capacity to create, receive, or transmit facts through a communications frequency. Consequently, this paper mainly claims to contribute a novel model to extend the period (time span) in intelligent systems based on the fuzzy and expert systems. We provide the conclusion of the current studies, models, and architectures concerning the new proposed model. More still, we provide a simplified mathematical representation of the model in perspective to vitality management and network period extension where presentation of the simulated results with disclosure of simulation parameters.

This paper possesses the following structure as tails. In section 2, related work per this study is provided. In section 3, the methodology of claimed model is brightened. In section 4, the results and their technical discussion are explicated. Section 5 holds the paper's conclusion is presented also lastly with indistinctly depicting our future research.

\section{Literature Review}

Within this section entails the state-of-the-art related studies that pertain to vitality consumption minimization about response time depicting the gap to carry out this study. To begin with, the designed model in dissimilarity of optimized hybrid energy-competent dispersed etiquettes named as tier chaining and ICHBFL- grounded Tier chaining protocols were proposed to develop amalgam vitality- efficient distributed protocol is one of the prominent clustering conventions in WSNs leading to utilization their vitality possessions competently and provided that permanency to network nevertheless the model didn't cogitate the detachment from the sensors into the sink well detailed [11].

Another option that has been proposed is the deployment of additional or supplementary nodes within extents nearer to the sink. Several studies for example as demonstrated in [12] wished-for a 
non-unvarying node circulation stratagem that splits the sensing turf hooked on numerous aureoles also elasticity the proportion in knot solidities between two sequential aureoles. The identified challenge to this approach or model is that the model has exponential evolution of the overall quantity of node measuring devices within the network. Additionally, the zone round the sink might have numerous nodes, the sink may be critical for a MAC complied etiquette to regulate the admittance of these protuberances to the channel or to undertake their duty rotations wireless-ly.

The studies additionally to the above-suggested model, in [13] proposed a novel non-uniform node circulation approach grounded on their examination and a channeling procedure joined with the projected node scattering approach for accomplishing the suboptimal dynamism effectiveness. The domino effect depicted that in case the net epoch ends, the inner auras approximately accomplish balanced vitality depletion over the model aspects the same challenges as early suggested in [7].

To deliver appreciate sensing, coordinating mechanisms are vital amongst actors among other traits. Another way to prolong the net period to attain node mobility in mobile WSNs leading to the balanced vitality depletion, likewise considering time sensor data is hypothetically must to be valid at the period of acting. The mobility of sensor nodes is feasible with the capacity to accomplish in diverse ways as illustrated in [14]. The study demonstrated further showing that sensors may most of the times is fortified mobilizing and shifting their identifiable position. The model may be categorized per a) mobile element setup, b) sink mobility which is sometimes referred to as a mobile relay that proposed scheme belongs to the mobile sink detailed in [15].

A solution to the mobile sink challenges in hierarchical structure sensor net was proposed in [16]. The authors begin with the locomotive sinks starting at a constant location of technical path of sink leading back to the first identical location. Occasionally, the nodes with sensing capabilities are casually situated or mounted in the net atmosphere. A constellation head wrinkles info from the protuberances in its bunch, redeemable data within the cushion, and lastly transmit the files to the locomotive sinks in case it is contracted at desirable level where vitality draining is minimal is depletion.

Furthermore, the authors introduced a vitality solution model shorthanded as GMRE in [17]. Basing on GRME, the locomotive sinks choses a new position that is surrounded by nodes of increased and great remaining energies. To attain info concerning the remaining energies, unique sentinel nodes are some chose around ever possible environment. Conditions may involve the vitality data as adjacent nodes to the locomotive nodes. In addition to the above, an alternative metaheuristic per relocation model is used [18], where still mobile sinks select it is better position that are proximately close the nodes with the sophisticated live and selective sparse traffic pattern.

Throughout visitations to nodes, the sink holds position to the node position for some moment. Nodes tends to negatively cover area close to the sink may and might send messages of multiple path-hop leading to put into the mobile sinks and contained by the use recommendable path in the shortest path possible routing. The studies derived a formulae that the optimum period of every sink environment. A similar approach, exploiting multiple mobile sinks is well detailed in [19].

\section{Methodology}

Within the section, the proposed model is presented, a traditional approach to vitality consumption minimization relation to with fuzzed network model.

\subsection{Tradition approach to Vitality efficiency}

Traditionally, to reduce the vitality in the network, different studies have been published in an attempt to have optimized vitality consumption. Consider a normal node partaking a customary of $N$ node $F=\{1, \ldots \mathrm{N}\}$. This is denoted by the $e_{i}$ for the static vitality and $\psi_{i}^{s}$ is as the dynamic vitality within the node that is intended to process nonnegative portions. $\forall_{i}$ expresses the input rate from the users' workload using the utilization of its local resources.

The ${ }^{\varpi_{i}}$ contains all computation rates and $\forall_{j}$ is constant in this method that caters to remaining workloads, in case any, is forwarded to the cloud for more complete execution. A dynamic power or 
vitality utilization is often the leading to the circuit activity and is examined through computing resources activities.

$$
\psi_{i}=e_{i}\left(\psi_{i}^{\delta}+\psi_{i}^{d} \varpi_{i} \forall_{i}\right)
$$

A node tends to partake in diverse workloads during the arrival rates. Therefore, to optimize the optimum reaction period of users associating with the whole supportive net nodes.

$$
n_{i}\left(\varpi_{i}\right) \frac{\psi_{i}}{\varpi_{i} \forall_{i}} e_{i}\left(\frac{\psi_{i}}{\varpi_{i} \forall_{i}}+\psi_{i}^{d}\right)
$$

There has been notation of the optimal period of fog node $i$ under $n_{i}$ is the power efficiency on the nodes, $\forall_{i}$ to be the total consumption of the power in the fog node for a given specified time of execution. Notably, $\forall_{i}$ where the node ${ }^{j}$, the received computation might be processed by network node. The workload $\forall_{i}$ of the computation done by the cloud. The response time associated with a given network node $i \quad R_{j}^{w i}$ is the response time of $j$, The workload processed locally Round Trip Time (RTT) where the devices in the network $\xi^{u}{ }_{j}$ in network or cloud serve are fixed. $\beta_{j}^{w i}=\zeta_{j}^{u}+\zeta^{f}$ depicting that computation was done in the cloud.

$$
\beta_{j}^{\psi 2}=\left(\forall_{i}\right)=\zeta_{i}^{s}+\frac{1}{\eta_{j}-\forall_{j}}
$$

In case, $\forall_{j} \leq \eta_{j}$ the need to analyze performance raises. ${ }^{\alpha_{j}}$ a portion of the workload of the node ${ }^{j}$ is at times partially in the network and the rest within the computations of the cloud. This leads to the constrains that $0 \leq \alpha_{j} \leq 1$ by the node ${ }^{j}$, then processed one in the cloud is ${ }^{\sigma_{j}-1}$. Therefore, the need to have $\alpha_{j} \forall_{i} \leq \eta_{j}$ is to obtain and satisfy equation 4 .

$$
\varpi^{*}=\underset{\arg u \min }{ } \eta_{i}\left(\varpi_{i}\right)
$$
$\beta_{i}\left(\varpi_{1}\right) \leq \overline{\beta_{i}} 0 \leq \varpi_{i} \leq 1, \forall_{\mathrm{i} \in \mathrm{F}}$.

Given that, the $c$ is the cooperation and $F$ which constrain below

$$
\overrightarrow{\vec{\sigma}}=\underset{\vec{\varpi}}{\arg \min } \sum_{i=F} \eta_{i}^{c}(\vec{\varpi})
$$

Alternatively, considered the assumption that a WSN entailing of N nodes with sensing ability and one mobile sink, those sensor nodes collect files from the locality location and use multihops in forwarding to supply data to the closer mobile entities as shown in [20]. Also considered further assumed the transmission diameter a case in point a disk, of sensor nodes is positioned in this model demonstrated [21], this contributes to the efficiency of algorithm established on multiclassifiers [22], ensuring the two-stage fuzzy statistics envelopment exploration [23], and applicability and performance evaluation of models as detailed in [24]. We considered some additional assumptions as listed below:

- The sink partakes satisfactory clout distribution of nodes is battery-motorized successions per limited vitality.

- All the nodes have similar operation characteristics like the vitality of batteries, a range of radio coverage among many more.

- Nodes are constant with at least one route, comprising of links wirelessly to the sink.

- Every sensor node, the remaining vitality, and the data cohort rate are acknowledged.

- The position of a node may be obtained using the GPS or other Position estimation techniques. 
- All estimations are done by the node itself and in the form of a special packet send to the sink.

- The first sensor node ${ }^{s_{i}}$ dies or, forgiven location $\Xi$, remaining vitality ${ }^{r e_{i}}$, with the data transmission rate $d t_{i}$ is denoted. Considering values of $\Xi r e_{i}$, and $d t_{i}$ is determined by $S_{i}$

\subsection{Fuzzy sink mobility scheme}

In the proposed method, the sensing field is separated into numerous regions and the central point of all regions select as a candidate location set, that new location of the mobile sink can be selected among this set. These regions that (we select or intend to select) is enough appropriate that assurances select among candidate location set is the adjacent optimal verdict for the new location of mobile sinks.

In the proposed scheme, we habit a fuzzy system for decision making about the new location of the mobile sink. Finally, the region with the highest score has the most priority to be chosen as the next position of the mobile sink; we further demonstrate how to compute the inputs of the system.

First, considering a timer $\Theta_{1}$ in each node. At intervals, every node provides a special control packet and directed to $\mathrm{t} h$ e sink.

This packet contains values of node traffic $\left({ }^{n t r} r_{i}\right)$, remaining vitality of node $\left({ }^{r e_{i}}\right)$ and node

locations $(\Xi)$. There is another timer $\Theta_{2}$ in the sink. At given intervals, $\Theta_{2}$ that is to say $\Theta_{2}=1.5 \Theta_{1}$, sink using the values of received control packets calculate the inputs of the fuzzy system. At first, according to the value of $\Xi$, sink estimate the number of node in each region $\left({ }^{\text {enum }_{j}}\right)$, then average traffic $(n t r)$ and average remaining vitality $(r e i)$ of each region is calculated.

In deduction to this, we regularize these values and use them as inputs of the fuzzy system and compute the score of each region as a candidate new location of the mobile sink.

$$
n t r=\frac{\sum n t r_{i}}{\text { enum }^{j}}
$$

Where $S_{i}$ is within the region $j$

$$
r e_{i}=\frac{\sum r e_{i}}{\text { enum }_{j}}
$$

Where $S_{i}$ is within the region $j$

Normalization means that every variable is normalized in the same range. For instance [0, 1]. A fuzzy expert system has mainly four parts namely fuzzifying point, interface engine point, Rule Base 
fuzzy-based point, defuzzying point, all these four parts are illustrated in Fig.1 as the proposed architecture claims.

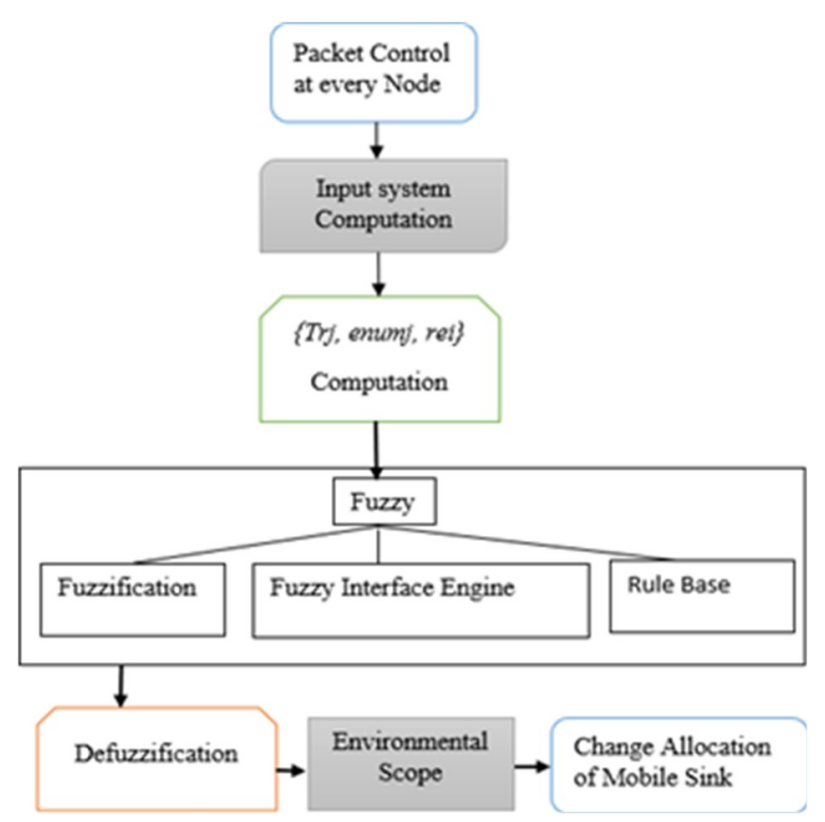

Figure 1. Proposed System Architecture

Each input crisp variable content allows initially to be undertaken under fuzzification to linguistic content in search for the decision making of fuzzy system processes per the rule base. Suppose that $\hbar=\left\{\hbar_{1} \hbar_{2} \hbar_{3}, \ldots . ., \hbar_{n}\right\}$ is the problem domain of the fuzzy model, fuzzification is responsible for maps $\hbar$ into the interval [0 1] using a membership function ${ }^{\mu}(\hbar)$.We habited a triangular fuzzification sine it is commonly used MF that is shown in Eq.(8), the MF for inputs of our system is illustrated in Fig. 2 below.

$$
\begin{aligned}
& \mu(\hbar)=\text { Triangular }(\hbar ; x, y, c) \\
= & \operatorname{Max}\left(\operatorname{Min}\left(\frac{\hbar-a}{y-x}, \frac{c-\hbar}{c-y}\right), 0\right)
\end{aligned}
$$

Commonly, the information ignoble vittles the rules that are rummage-sale by the uncertain extrapolation locomotives to get a newfangled datum from them. For the logic structure, it routines ambiguous IF-THEN rules as summarized in Table 1.

Table 1. Rules in Fuzzy Mobility Sink Scheme

\begin{tabular}{cccc}
\hline IF & & & THEN \\
\hline- & - & - & $y$ \\
Low & Low & - & 0 \\
Low & - & Low & 0 \\
\hline
\end{tabular}




\begin{tabular}{llll}
\hline Low & Med & Med & .12 \\
Low & Med & High & .25 \\
Med & Med & High & .63 \\
Med & High & Med & .5 \\
Med & High & High & .76 \\
High & Low & Low & .13 \\
High & Med & High & .88 \\
High & High & Low & .12 \\
High & High & Med & .63 \\
High & High & High & 1 \\
\hline
\end{tabular}

We furthermore applied Sugeno-style fuzzy inference which does not require defuzzification at all levels. In the proposed system, And the method is a T- norms such as min, or Prod. According to the value of $\hbar$ each input and membership function, the degree of compatibility is calculated. Then using Prod and degree of compatibility values, firing Strength ( $\varpi_{i}$ ) attained. Implication method is multiplication and the final outputs are accomplished from the following equation:

$$
y=\frac{\sum \varpi_{i} \cdot y i}{\sum \varpi_{j}}
$$

Finally, the final output as a score of regions and the region with the highest score is chosen as a new location and the mobile sink will change his location there.

\section{Results and Discussion}

In this section, analysis, and comparison in the performance of the proposed scheme that we named it FMS are presented. The simulation was done in the NS-3 simulator. Note that within the simulation, nodes are separated into sensor nodes and mobile sink node, and then numerous properties supplementary added to each as detailed below:

- In every node add one filed as a node type whereby the ordinary node is distinct from the sink.

- In sensor nodes add timer as a Scheduler for sending control data and one function to generate control packet and send it to mobile sink. 


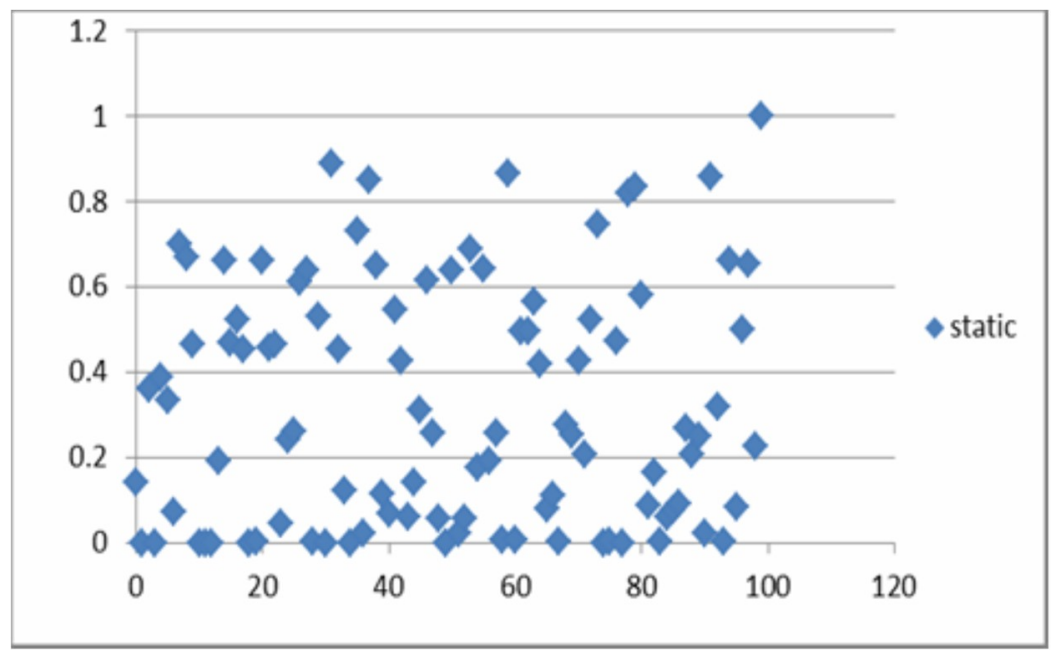

Figure 2a. Obtainable Energy of Nodes in Static Sink Schemes

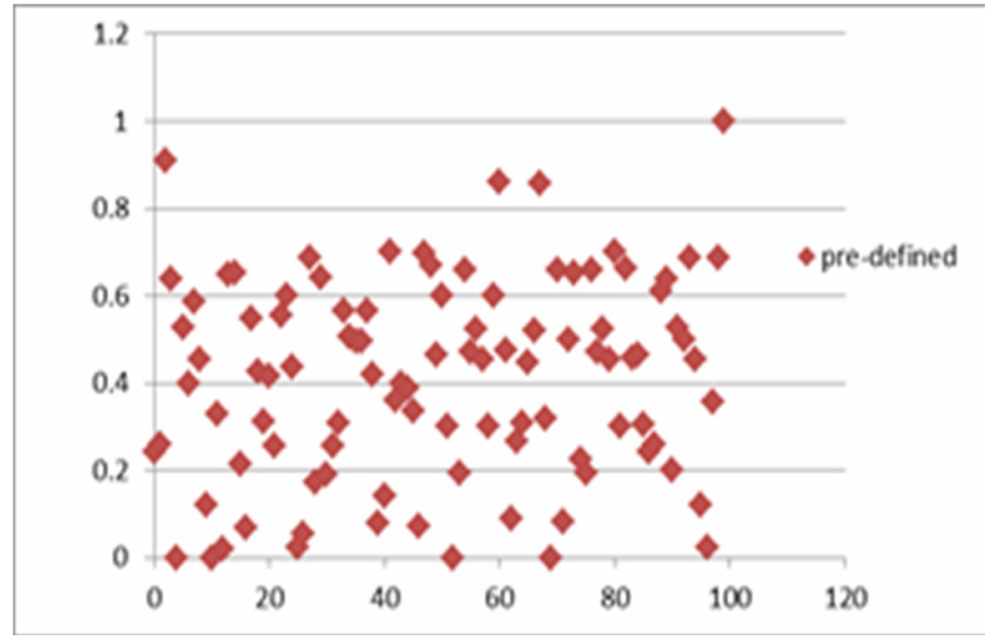

Figure 2b. Obtainable Energy of nodes in pre-defined sink mobility

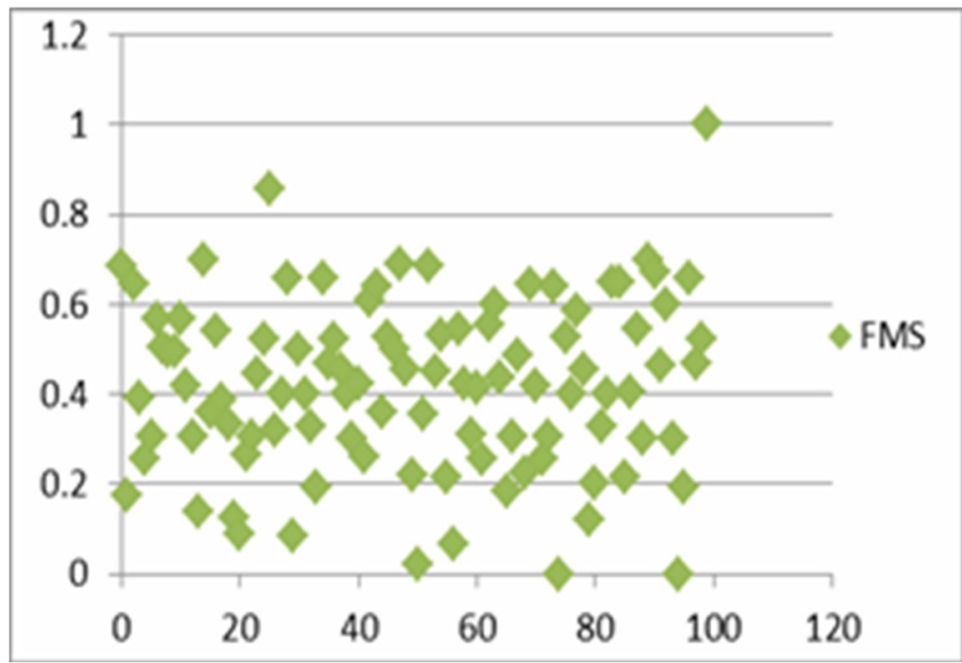

Figure 2c.Obtainable Energy of Nodes in Fuzzy Mobility Sink Scheme 


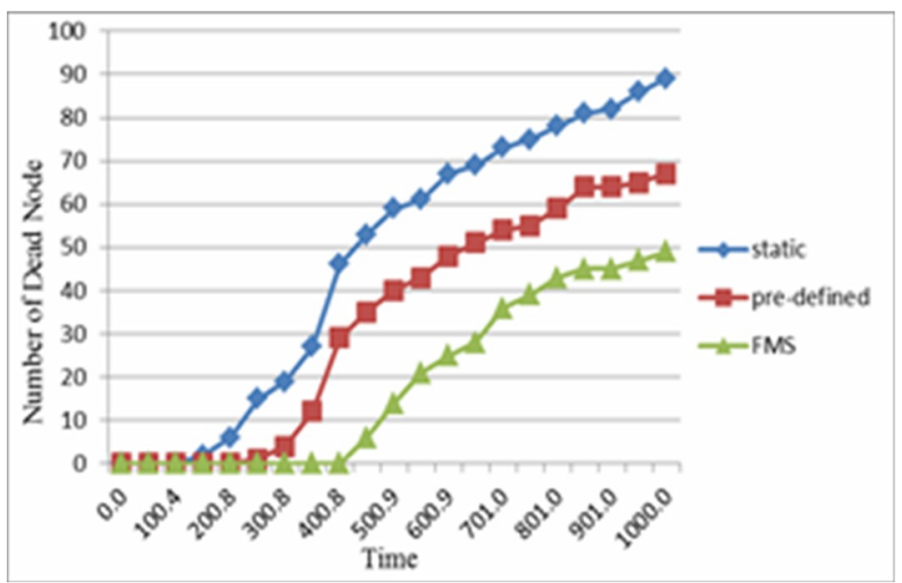

Figure 2d. Obtainable Number of Dead Nodes

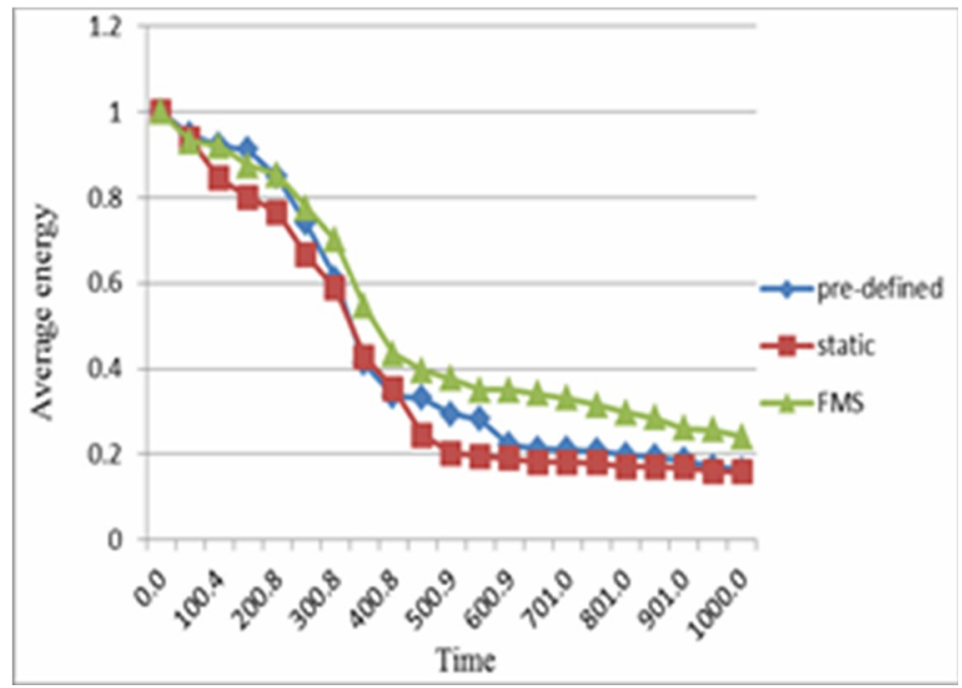

Figure 2e. Obtainable Average Residual Energy

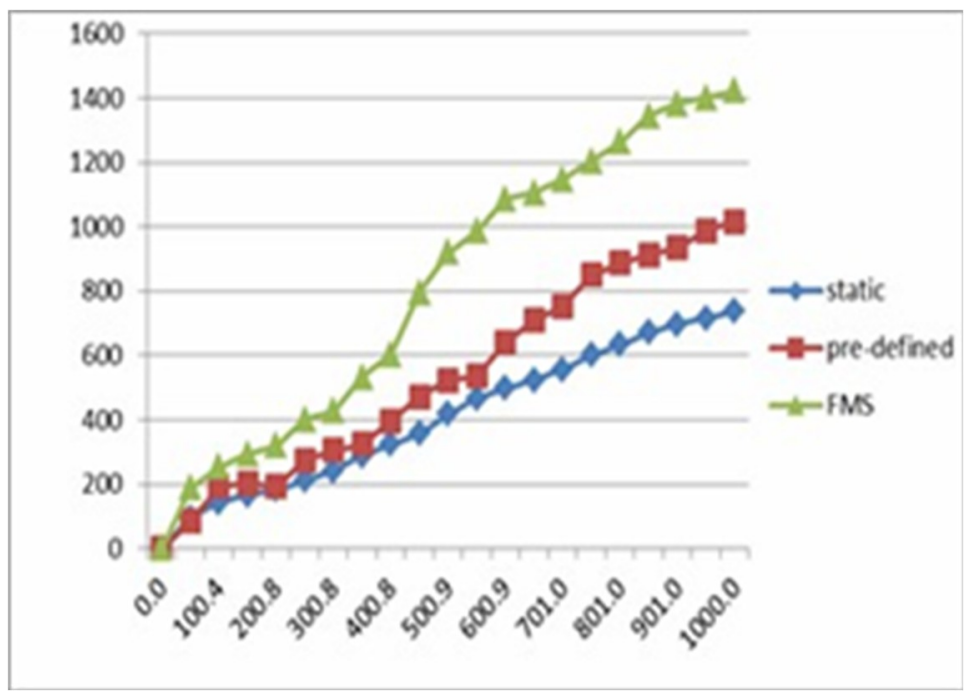

Figure 2f. Obtainable Presentation of the network overhead

The sensors are deployed randomly in the area and considered utilizations of appreciate parameters of the channel. Used values for the parameters are given in Table 2. The proposed model 
in this study was compared two other schemes that is to say: a static model where the sink node is static, a mobile that sinks follow a pre-defined path for collecting data from sensor nodes.

From the all illustrated illustrations in figure 1 above, we assessed and compared the proposed scheme (model) with the mentioned methods base on four criteria that each is represented network period (network period). Fig. 2 (e) show average remaining vitality in the network. As it can be notified, the newly proposed method leads to higher average vitality in network based on that one in comparison. There is higher average vitality implying that there is better performance in vitality conservation, nonetheless important to note that this is also not enough separately. Balanced vitality consumption in different parts of the network is an alternative criterion alongside the higher average remaining vitality that effects on network period. In this same line, we further used a variance of vitality consumption that demonstrates the remaining vitality of all the nodes in half-time of simulation. The fig. $2 \mathrm{a}, 2 \mathrm{~b}$, plus $2 \mathrm{c}$ confirm all claims that were early stated about the scheme energy consumption is more balanced and vilified.

From the all illustrated illustrations in figure 1 above, we assessed and compared the proposed scheme (model) with the mentioned methods base on four criteria that each is represented network period (network period). Fig. 2 (e) show average remaining vitality in the network. As it can be notified, the newly proposed method leads to higher average vitality in network based on that one in comparison. There is higher average vitality implying that there is better performance in vitality conservation, nonetheless important to note that this is also not enough separately. Balanced vitality consumption in different parts of the network is an alternative criterion alongside the higher average remaining vitality that effects on network period. In this same line, we further used a variance of vitality consumption that demonstrates the remaining vitality of all the nodes in half-time of simulation. The fig. $2 a, 2 b$, plus $2 c$ confirm all claims that were early stated about the scheme energy consumption is more balanced and vilified.

\section{Conclusion}

This study claimed and proposed a new mobile sink tactic based on fuzzy logic and intelligent systems like an expert system that chooses the finest location in-network location for the mobile sink as an extending the network period. It has been revealed that unstable vivacity ingesting in diverse portions of the environment in WSNs is still the main limitation for network period or network period. The provided simulation results demonstrated the proposed model suggestively prolong network period compared to the models listed in section 2. We are hoping to work shortly on enthusiastically determine a set of candidate locations for the mobile sink, instead of dividing the network into different regions, also we want to improve our fuzzy system and combine it with multiple mobile sink scheme in approach to improve network retro.

\section{Data Availability}

The data used to support the findings of this study are included within the article.

\section{Disclosure}

The research was performed extracted from the thesis of first author as a part of academic interest of the first author and was carried out at Yazd University, Yazd, Iran.

\section{Conflicts of Interest}

The authors declare that there are no conflicts of interest regarding the publication of this paper.

\section{Funding Statement}

This study is been supported fully by the computer Engineering Department of Yazd University of Yazd University. 


\section{Acknowledgements}

The authors acknowledge the department of Computer Engineering for extending their important comments and suggestion that helped us to produce the quality of paper.

Author Contributions: The authors have contributed equally. Both authors contributed in conceptualization, methodology, simulation software or tools, validation, formal analysis, topic investigation, resources, data curation, writing-original draft preparation, writing review and editing

\section{References}

1. G. Pau and V. M. Salerno, "Wireless Sensor Networks for Smart Homes: A Fuzzy-Based Solution for an Vitality-Effective Duty Cycle," Electronics, vol. 8, no. 2, p. 131, 2019.

2. P. K. H. Kulkarni and P. M. Jesudason, "Multipath data transmission in WSN using exponential cat swarm and fuzzy optimization," IET Commun., vol. 13, no. 11, pp. 1685-1695, 2019.

3. T. AZFAR, W. AHMED, A. HASEEB, R. AHMAD, S. T. GUL, and F.-C. ZHENG, "A low complexity online controller using fuzzy logic in vitality harvesting WSNs," Sci. CHINA Inf. Sci., vol. 62, no. 4, p. 042305, 2019.

4. W. Peng, C. Li, G. Zhang, and J. Yi, "Interval type-2 fuzzy logic- based transmission power allocation strategy for period maximization of WSNs," Eng. Appl. Artif. Intell., vol. 87, p. 103269, 2020.

5. Y. Chittibabu, C. H. Anuradha, and S. R. C. P. Murty, "Fuzzy Trust-Based Vitality-Aware Multipath Secure Data Collection in Wireless Sensor Network," J. Comput. Theor. Nanosci., vol. 16, no. 2, pp. 669-675, 2019.

6. S. Tabatabaei, A. Rajaei, and A. M. Rigi, "A Novel Vitality- Aware Clustering Method via Lion Pride Optimizer Algorithm (LPO) and Fuzzy Logic in Wireless Sensor Networks (WSNs)," Wirel. Pers. Commun., pp. 1-23, 2019.

7. M. S. Tomar and P. K. Shukla, "Vitality Efficient Gravitational Search Algorithm and Fuzzy Based Clustering With Hop Count Based Routing For Wireless Sensor Network," Multimed. Tools Appl., pp. 1$22,2019$.

8. S. K. Mothku and R. R. Rout, "Adaptive Fuzzy-Based Vitality and Delay-Aware Routing Protocol for a Heterogeneous Sensor Network," J. Comput. Netw. Commun., vol. 2019, 2019.

9. A. Mahboub, E. M. En-Naimi, M. Arioua, H. Barkouk, Y. El Assari, and A. El Oualkadi, "An vitalityefficient clustering protocol using fuzzy logic and network segmentation for heterogeneous WSN.," Int. J. Electr. Comput. Eng. 2088-8708, vol. 9, 2019.

10. P. Gupta and A. K. Sharma, "Clustering-based Optimized HEED protocols for WSNs using bacterial foraging optimization and fuzzy logic system," Soft Comput., vol. 23, no. 2, pp. 507-526, 2019.

11. X. Wu, G. Chen, and S. K. Das, "On the vitality hole problem of nonuniform node distribution in wireless sensor networks," in 2006 IEEE International Conference on Mobile Ad Hoc and Sensor Systems, 2006, pp. 180-187.

12. W. X.-B. C. Gui-Hai, "The vitality hole problem of nonuniform node distribution in wireless sensor networks [j]," Chin. J. Comput., vol. 2, no. 010, 2008.

13. I. F. Akyildiz and I. H. Kasimoglu, "Wireless sensor and actor networks: research challenges," Ad Hoc Netw., vol. 2, no. 4, pp. 351-367, 2004.

14. Y. Yang, M. I. Fonoage, and M. Cardei, "Improving network period with mobile wireless sensor networks," Comput. Commun., vol. 33, no. 4, pp. 409-419, 2010.

15. E. M. Saad, M. H. Awadalla, and R. R. Darwish, "A data gathering algorithm for a mobile sink in largescale sensor networks," in 2008 The Fourth International Conference on Wireless and Mobile Communications, 2008, pp. 207-213.

16. S. Basagni, A. Carosi, E. Melachrinoudis, C. Petrioli, and Z. M. Wang, "Controlled sink mobility for prolonging wireless sensor networks period," Wirel. Netw., vol. 14, no. 6, pp. 831-858, 2008.

17. K. Akkaya and M. Younis, "Vitality-aware routing to a mobile gateway in wireless sensor networks," in IEEE Global Telecommunications Conference Workshops, 2004. GlobeCom Workshops 2004., 2004, pp. 1621.

18. W. Alsalih, H. Hassanein, and S. Akl, "Placement of multiple mobile data collectors in underwater acoustic sensor networks," Wirel. Commun. Mob. Comput., vol. 8, no. 8, pp. 1011-1022, 2008.

19. R. Sharma, V. Vashisht, and U. Singh, "EEFCM-DE: vitality- efficient clustering based on fuzzy C means and differential evolution algorithm in WSNs," IET Commun., vol. 13, no. 8, pp. 996-1007, 2019. 
20. W. Shafik and S. M. Matinkhah, “Admitting New Requests in Fog Networks According to Erlang B Distribution," in 2019 27th Iranian Conference on Electrical Engineering (ICEE), 2019, pp. 2016-2021.

21. G. Büyüközkan, C. A. Havle, O. Feyzioğlu, and F. Göçer, “A combined group decision making based IFCM and SERVQUAL approach for strategic analysis of airline service quality," J. Intell. Fuzzy Syst., vol. 38, no. 1, pp. 859-872, 2020.

22. D. Yildiz, G. T. Temur, A. Beskese, and F. T. Bozbura, “Evaluation of positive employee experience using hesitant fuzzy analytic hierarchy process," J. Intell. Fuzzy Syst., vol. 38, no. 1, pp. 1043-1058, 2020.

23. A. Kaya, B. Çiçekalan, and F. Çebi, "Location selection for WEEE recycling plant by using Pythagorean fuzzy AHP," J. Intell. Fuzzy Syst., vol. 38, no. 1, pp. 1097-1106, 2020. 